Cahiers de philosophie de l'université de

\title{
La notion de " communauté » chez Max Weber : enjeux contemporains
}

Catherine Colliot-Thélène

\section{OpenEdition}

1 Journals

Édition électronique

URL : https://journals.openedition.org/cpuc/425

DOI : $10.4000 /$ cpuc. 425

ISSN : 2677-6529

Éditeur

Presses universitaires de Caen

Édition imprimée

Date de publication : 30 septembre 2019

Pagination : 35-56

ISBN : 978-2-84133-942-6

ISSN : $1282-6545$

Référence électronique

Catherine Colliot-Thélène, "La notion de « communauté » chez Max Weber : enjeux contemporains », Cahiers de philosophie de l'université de Caen [En ligne], 56 | 2019, mis en ligne le 30 septembre 2020, consulté le 31 janvier 2023. URL : http://journals.openedition.org/cpuc/425 ; DOI : https://doi.org/ 10.4000/cpuc. 425

\section{(c) (7) (5)}

Creative Commons - Attribution - Pas d'Utilisation Commerciale 4.0 International - CC BY-NC 4.0 https://creativecommons.org/licenses/by-nc/4.0/ 


\title{
La notion de "communauté» chez Max Weber : enjeux contemporains
}

\begin{abstract}
[...] l'image d'un monde fini (la planète Terre) comme cadre englobant, homogénéisant, dépassant les contextes locaux ou nationaux, a mis en question le périmètre de ce qui «nous» est commun, allant des classiques «communautés villageoises » ou «communautés ethniques » à la « communauté européenne», voire à la «communauté internationale» celles-ci se révélant moins évidentes que celles-là, avant tout pour des raisons d'échelle de grandeur et donc d'intensité de communication «interne». Le mot «communauté» s'est alors trouvé remis en scène et en question. Dans le débat public, il est devenu pratiquement équivalent à celui d'identité. «Identité» s'impose à « communauté» et en surdétermine le sens dans les contextes généralement nationaux des sociétés complexes.

Michel Agier, La condition cosmopolite ${ }^{1}$
\end{abstract}

\section{Communauté, communautisation et sociétisation chez Max Weber}

Dans la brève remarque préliminaire sur laquelle s'ouvre l'édition classique d'Économie et société, Max Weber fournit quelques indications très succinctes sur ses sources ${ }^{2}$. Il mentionne notamment «le beau livre de Ferdinand Tönnies», Gemeinschaft und Gesellschaft, sans commentaire

1. M. Agier, La condition cosmopolite. L'anthropologie à l'épreuve du piège identitaire, Paris, La Découverte, 2013, p. 103.

2. M. Weber, Économie et société I, trad. par un collectif sous la direction d'E. de Dampierre, Paris, Plon, 1971, p. 3. - Il s'agit plus précisément d'une remarque préliminaire au chapitre premier, «Les concepts fondamentaux de la sociologie». Nos références vont dans la suite à la traduction nouvelle faite de ce texte par Jean-Pierre Grossein, sous le titre Concepts fondamentaux de sociologie (Paris, Gallimard [Tel], 2016). 
particulier. Quand il y revient, dans une remarque accompagnant le paragraphe 9 des "Concepts fondamentaux de sociologie», c'est pour faire état d'une différence importante entre sa conceptualisation et celle de Tönnies. Il ne parlera pas en effet de «communauté» et de «société», mais de «communautisation» et de «sociétisation». Tout en reconnaissant que cette distinction rappelle celle qui fait le titre de «l'ouvrage fondamental» de Tönnies, il note que celui-ci a donné à sa distinction, pour des fins qui lui étaient propres, «un contenu nettement plus spécifique» que le contenu que lui-même entend donner à l'opposition entre "communautisation" et "sociétisation " ${ }^{3}$. Il n'en dit pas plus, laissant à son lecteur la charge de préciser le point de la différence.

Ce qui frappe tout d'abord ce lecteur est la substitution de termes désignant des procès aux catégories substantielles de Tönnies. Weber n'oppose pas deux types de collectifs structurellement distincts, mais deux modes de formation des collectifs, dont il remarque d'ailleurs qu'ils sont souvent mêlés dans la réalité: «la grande majorité des relations sociales, note-t-il, revêt en partie le caractère d'une communautisation, en partie celui d'une sociétisation ${ }^{4}$. Mais il est une autre différence entre les conceptualisations de Tönnies et de Weber, dont on peut supposer que c'est ce à quoi Weber fait allusion quand il évoque leurs "fins» respectives. La distinction typologique entre, d'un côté, la Gemeinschaft (communauté), forme de collectif reposant sur un sentiment affectif d'appartenance partagé par tous les membres de ce collectif, et, de l'autre côté, la Gesellschaft (société), relation contractuelle ou quasi contractuelle entre individus, dépourvue de toute dimension affective, supportait chez Tönnies une philosophie de l'histoire: l'histoire de la civilisation moderne était interprétée, en gros, comme un passage de la communauté à la société. Sur ces significations typologique et historique se greffait en outre une intention politique: Tönnies était socialiste, et, bien que son concept de la communauté ait été construit sur l'exemple des sociétés agraires prémodernes, il pensait possible de faire revivre dans une société à venir, industrielle et urbaine, l'éthos de la communauté.

La version que j'ai dite «classique» d'Économie et société a été composée après la mort de Max Weber en rassemblant des textes dont les dates de rédaction s'échelonnent sur plus de dix ans. Ces textes avaient été conçus

3. M. Weber, Concepts fondamentaux..., p. 143. - Tous les traducteurs de Max Weber en français ont dû recourir à des néologismes pour rendre les termes Vergemeinschaftung et Vergesellschaftung. Pour notre traduction des Communautés, Élisabeth Kauffmann et moi-même (voir infra) nous sommes arrêtées à "communautisation» et "sociétisation", suivant en cela Jean-Pierre Grossein (voir le glossaire raisonné de sa traduction des Concepts fondamentaux, p. 332-333).

4. M. Weber, Concepts fondamentaux..., p. 143. 
pour être la contribution personnelle de Weber à une entreprise collective, une encyclopédie des sciences économiques et sociales, annoncée sous le titre Grundriß für Sozialökonomik [Fondements de l'économie sociale], dont Weber assumait par ailleurs la responsabilité éditoriales. L'édition critique de l'ensemble de l'œuvre de Max Weber (Max Weber-Gesamtausgabe), commencée en 1984 et tout récemment achevée, a redistribué et publié séparément les différents manuscrits réunis dans Économie et société. L'un des volumes résultant de cette redistribution s'intitule Gemeinschaften $^{6}$ [Communautés] : on y trouve, dans des versions amendées, des textes qui avaient été intégrés dans la seconde partie de l'édition parue en traduction française en 1971, et quelques annexes jusqu'alors non publiées. Une traduction française de ce texte (par Élisabeth Kauffmann et moimême) est parue en 2019 aux éditions La Découverte7 . La conceptualisation en est sensiblement différente de celle des textes plus tardifs, et notamment des Concepts fondamentaux de sociologie.

Weber utilise déjà, dans les manuscrits des Communautés, les termes Vergemeinschaftung et Vergesellschaftung, que nous avons donc rendus par "communautisation» et "sociétisation». Les deux termes ne désignent cependant pas encore deux types opposés de formation des collectifs sociaux: la sociétisation est ici une rationalisation d'une communautisation quelconque, c'est-à-dire son organisation en fonction de fins réfléchies. Weber emploie par ailleurs abondamment dans ce texte le terme Gemeinschaft, ce qui n'est plus le cas dans les Concepts fondamentaux de sociologie. Le premier manuscrit porte sur «les relations économiques des communautés en général», les quatre suivants, successivement, sur les communautés domestiques, les communautés ethniques, la communauté de marché, les communautés politiques. S’y ajoutent un manuscrit intitulé «Prestige du pouvoir et sentiment national» et un autre sur les «classes», "groupement statutaires» et les «partis» : c'est encore de types de communautés qu'il est question dans ces derniers manuscrits, celles qui sont constituées par le sentiment national, par les solidarités de classes, de statuts ou par les partis politiques. Lorsque Weber choisit

5. Pour être exacte, les éditeurs des premières versions de Wirtschaft und Gesellschaft ne se sont pas limités aux manuscrits conçus par Weber pour sa contribution au Grundriß Ils y ont également inclus des extraits de ses écrits politiques. - On trouvera quelques indications supplémentaires concernant les problèmes éditoriaux posés par Économie et société dans C. Colliot-Thélène, La sociologie de Max Weber, Paris, La Découverte, 2006, nouvelle édition 2014, p. 27-29.

6. Max Weber-Gesamtausgabe [désormais MWG] I/22-1, Tübingen, Mohr (Siebeck), 2001.

7. M. Weber, Les communautés, C. Colliot-Thélène et E. Kauffmann (éd. et trad.), Paris, La Découverte (Textes à l’appui. Série Politique et sociétés), 2019. 
de faire, en 1920, de la «relation sociale», et non plus de la «communauté», la catégorie clé des "concepts fondamentaux de sociologie», il rompait très consciemment avec sa conceptualisation des années 1910-1913, durant lesquelles (approximativement) avaient été rédigés les textes des Communautés.

À la fin de l'année 1913, Weber adressa à l'éditeur du Grundriß, Paul Siebeck, une lettre dans laquelle il annonçait ce que serait sa propre contribution. Il disait avoir élaboré une théorie qui «met en relation toutes les grandes formes de communautés avec l'économie, depuis la famille et la communauté domestique jusqu'à l'entreprise, le clan, la communauté ethnique, la religion (englobant toutes les grandes religions de la terre: une sociologie des doctrines de salut et des éthiques religieuses [...]); enfin, une théorie sociologique globale de l'État et de la domination $»^{8}$. La première partie de cette déclaration correspond assez bien aux manuscrits des Communautés. La mention des doctrines de salut et des éthiques religieuses ainsi que d'une théorie de l'État et de la domination indique toutefois déjà les chantiers de recherche nouveaux dans lesquels Weber s'était récemment engagé, qui l'ont amené à réviser la construction originaire qu'il avait prévue pour sa contribution au Grundriß et, finalement, à l'abandonner.

Je ne m'arrêterai pas cependant ici sur les raisons pour lesquelles Weber a finalement renoncé à faire de la notion de communauté le terme clé de sa contribution au Grundriß. Il va de soi que les exégètes de Weber doivent y porter attention. Mais, outre que le texte des Communautés a sa propre cohérence et mérite d'être lu pour lui-même, il présente un intérêt particulier à notre époque, dans la mesure où, comme le relève Michel Agier dans la citation placée en exergue de cet article, le mot «communauté» se trouve aujourd'hui « remis en scène et en question». Comme le constate Agier encore, ce mot est souvent utilisé, dans le débat public, comme un équivalent du terme «identité». Mais l'on perçoit aussi, dans des milieux savants, philosophiques et sociologiques notamment, une tendance récente à substituer le terme «commun» (employé comme substantif singulier) à la communauté. La publication en français des manuscrits de Weber sur les communautés nous donne l'occasion de nous interroger sur les usages, politiquement chargés, des termes "communauté», «identité» et "commun», hier et aujourd'hui. On verra que le texte de Weber présente, de ce point de vue, de remarquables potentialités critiques.

8. Lettre de Max Weber à Paul Siebeck du 30 décembre 1913, in MWG II/8, Tübingen, Mohr (Siebeck), 2003. 


\section{Communauté, identité, commun : hier et aujourd'hui}

Le terme «communauté» évoque aujourd'hui tout d'abord les débats du tournant du siècle entre les libéraux anglo-saxons (ou, en France, les républicains) et les communautariens. Bien que ce débat ne soit plus aussi central qu'il l'était dans les années 1980-1990 et au début des années 2000, on en trouve un écho dans les revendications que l'on qualifie aujourd'hui d' «identitaires». Le terme est avant tout utilisé pour désigner des mouvements politiques d'extrême droite, hostiles aux migrants et xénophobes. Il est pour cette raison généralement suspect du côté de la gauche et de l'extrême gauche politique. On assiste cependant depuis quelques années à une revalorisation généralisée de l'idée de nation, et, avec elle, des notions de communauté et d'identité nationale. Bien des apôtres du "post-national» et du cosmopolitisme des années 1990 et 2000 reconnaissent aujourd'hui la résistance des «identités nationales» et cherchent par exemple, s'agissant de l'Union européenne, à faire une place à la représentation des peuples nationaux dans les institutions de cette Union' 9 . Souvent aussi, ils acceptent le bien-fondé d'un des reproches que leur faisaient les adversaires du cosmopolitisme, qui était d'ignorer ou de sous-estimer l'importance des sentiments ou, plus généralement, du «symbolique», dans les processus de formation des collectifs sociaux et politiques.

Ce qui demeure des problèmes soulevés naguère par les communautariens est la question de l'appartenance: toute communauté, et la communauté politique comme toute autre, est-elle une forme d'appartenance, toujours spécifique par conséquent, impliquant que soit établie une délimitation entre un intérieur et un extérieur? L'identité (entendue comme caractère distinctif) de la communauté a-t-elle pour condition ou pour effet une identité (entendue comme similitude) de ses membres, et par conséquent une clôture, par rapport à «l'étranger», quelle que soit la manière dont il est déterminé? La réponse paraît ne pouvoir être que positive, et la plus grande partie des discussions porte en effet sur le type d'identité (raciale, religieuse, culturelle, citoyenne) sur lequel pourrait ou devrait reposer cette appartenance dans le cas des communautés démocratiques, ainsi que sur le caractère plus ou moins rigoureux de leur clôture. C'est en fonction de ces deux critères, le fondement de

9. C'est le cas en particulier de Jürgen Habermas dans Zur Verfassung Europas, Berlin, Suhrkamp, 2011 (en français: La constitution de l'Europe, C. Bouchindhomme [trad.], Paris, Gallimard [NRF essais], 2012). - Voir à ce propos mon analyse critique dans l'ouvrage collectif publié sous la direction d'Isabelle Aubert et Jean-François Kervégan, Dialogues avec Jürgen Habermas (Paris, CNRS Éditions, 2018, p. 45-56) ainsi que la réponse de Habermas dans le même ouvrage (p. 466-468). 
l'identité communautaire et la rigueur de sa clôture, que se distinguent et s'opposent les différentes variantes des nationalismes contemporains. De ce point de vue, l'humanisme cosmopolitique paraît un idéal abstrait, incompatible avec les conditions d'existence des communautés réelles. Il y a certes des différences considérables entre le nationalisme xénophobe d'un Viktor Orbán en Hongrie et une conception républicaine de la nation que certains défendent en France, aujourd'hui (en se réclamant éventuellement d'Émile Durkheim ou de Marcel Mauss). Mais aussi bien le nationalisme xénophobe que le républicanisme national invitent à se demander si le fondement des collectifs politiques réside effectivement dans une forme spécifique d' «identité», dont la nation reste aujourd'hui, et sans doute pour longtemps encore, un cas exemplaire.

Avant d'y revenir, je rappellerai brièvement deux autres moments de l'histoire politique de la notion de "communauté» au XXe siècle. Ce ne sont bien entendu que des jalons pour une histoire de la "communauté» qui reste à écrire, laquelle devrait notamment remonter jusqu'aux usages de ce terme dans le christianisme ${ }^{10}$.

Le premier moment est spécifiquement allemand, il concerne donc le terme Gemeinschaft, et il est déjà lointain. Il débute par la publication de l'ouvrage déjà cité de Ferdinand Tönnies, Gemeinschaft und Gesellschaft, dont la première édition date de 1887 . Le succès grand public du livre de Tönnies commence avec sa seconde édition, remaniée, en 1912, et il s'élargit avec sa troisième édition en 1920. Dès 1922, le sociologue Helmut Plessner constatait que «l'idole de notre temps est la communauté [Das Idol dieses Zeitalter ist die Gemeinschaft] " ${ }^{11}$. L'historien et politologue allemand Kurt Sontheimer (1928-2005) a pu écrire, dans un ouvrage publié en 1962 (Antidemokratisches Denken in der Weimarer Republik), que le terme "communauté» "fut l'un des mots magiques à l'époque de Weimar ${ }^{12}$. La distinction entre communauté et société était en effet devenue, sous Weimar, un lieu commun du débat intellectuel et politique, mais elle avait

10. L'entrée «Gesellschaft, Gemeinschaft» des Geschichtliche Grundbegriffe (Historisches Lexikon zur politisch-sozialen Sprache in Deutschland, Stuttgart, Klett-Cotta, vol. 2, 1975) ne donne que quelques indications succinctes à ce propos (p. 806-808). Il ressort de cette entrée que les termes societas et communitas étaient utilisés sans distinction nette dans la philosophie sociale de l'Antiquité tardive et du christianisme médiéval. Les auteurs de l'article notent cependant une préférence pour communitas (également communio et communicatio) dans le commentaire aristotélicien, chez Thomas d'Aquin, Albert le Grand, Guillaume d'Ockham et Marsile de Padoue.

11. H. Plessner, Grenzen der Gemeinschaft, Bonn, F. Cohen, 1924, cité d'après M. Wildt, Volk, Volksgemeinschaft, AfD, Hambourg, Hamburger Edition, 2017, p. 70.

12. Cité d'après O. Jouanjan, Justifier l'injustifiable. L'ordre du discours juridique nazi, Paris, PUF (Léviathan), 2017, p. 153 
perdu dans cet usage «toute la sophistication sociologique qu'elle avait chez Tönnies pour se réduire à une opposition sommaire de slogans ${ }^{13}$. La référence positive à la Gemeinschaft, et aussi l'usage positif de l'expression Volksgemeinschaft, se retrouvent sous la plume de la quasi-totalité des acteurs politiques de la République de Weimar, de droite comme de gauche. Chez les uns (libéraux et sociaux-démocrates), l'invocation de la communauté du peuple visait à l'inclusion: l'objectif était de mettre fin aux conflits violents qui déchiraient la société allemande dans les années 1920, en s'appuyant sur la Constitution de Weimar, garante de la liberté de tous. Pour les autres, représentants de l'extrême droite et idéologues nazis, la communauté du peuple devait être fondée sur l'homogénéité raciale, donc sur l'exclusion de l' "hétérogène», les juifs en tout premier lieu. La captation du terme Gemeinschaft par la langue du Troisième Reich pèse encore sur les possibilités de ses usages actuels en Allemagne. Dans le petit ouvrage incisif qu'il a consacré récemment au parti d'extrême droite allemand, l'Alternative für Deutschland (AfD), l'historien allemand Michael Wildt critique les tentatives faites par certains des représentants de ce parti pour réhabiliter notamment l'expression Volksgemeinschaft (communauté du peuple). «Après les crimes du régime national-socialiste, écrit-il, qui ont été commis au nom de la Volksgemeinschaft, il n'y a plus d'usage "innocent" de ce concept ${ }^{14}$. Le terme Gemeinschaft lui-même, dont il est de nombreux emplois spécifiques, ne souffre pas d'un même tabou. Il est cependant utilisé avec parcimonie dans les discours politiques et les textes de théorie politique. Il est absent, notamment, des constructions théoriques élaborées par les deux plus célèbres représentants de la théorie critique de ces dernières décennies, Jürgen Habermas et Axel Honneth.

Un moment plus récent de l'usage politique du terme «communauté » est français et italien. À la différence de celui précédemment évoqué, il s'est joué exclusivement dans le milieu restreint de la philosophie. Il est jalonné par quelque ouvrages, dont je cite ici les titres: Jean-Luc Nancy, La communauté désœuvrée, 1983 (1986, 1999), qui était un commentaire de Georges Bataille; Maurice Blanchot, La communauté inavouable, 1983; Nancy encore, La communauté affrontée, 2001; La communauté désavouée, 2014; Giorgio Agamben, La comunità che viene, 1990 (traduit en français la même année: La communauté qui vient), auxquels il faut ajouter les allusions à la première phase de ce débat chez Derrida, dans une longue note de Politiques de l'amitié15. Et encore: Roberto Esposito, Communitas:

13. O. Jouanjan, Justifier l'injustifiable..., p. 153.

14. M. Wildt, Volk, Volksgemeinschaft, AfD, p. 116.

15. J. Derrida, Politiques de l'amitié, Paris, Galilée (La philosophie en effet), 1994, p. 56-57. 
origine et destin de la communauté, 200o. Disons-le clairement: ces textes, très ésotériques, n'apportent pas grand-chose à une réflexion sur les usages politiques possibles de la notion de communauté. Ils ont cependant une signification "politique», parce qu'ils ont été motivés à l'origine par l'échec du «communisme réel». Nancy le constate avec lucidité dans un texte de 2014 où il revient sur son débat avec Blanchot dans les années 1980. Il s'agissait, écrit-il, «d'interroger le sens ou la teneur d'un mot tel que "communauté" qui ne proposait en substance rien d'autre que "communisme" sans le discrédit politique dans lequel ce dernier était tombé» ${ }^{16}$. Mais si le contexte de cette réflexion sur la communauté était incontestablement politique (la fin de l'espérance communiste), le résultat était très frustrant. Comme Nancy le note encore dans son texte de 2014, il avait cherché chez Bataille une politique, mais il n'y avait trouvé qu'un renoncement à la recherche d'une communauté politique ${ }^{17}$. Le terme "communauté» avait cependant en français une «innocence» que ne pouvait avoir en Allemagne le terme Gemeinschaft. Il était possible, en France, de tenter de penser sous ce terme une forme de socialité accomplie, même si la démarche aboutit en définitive à une aporie: «l'absence de tout antagonisme sérieux à la civilisation déterminée par le capitalisme ${ }^{18}$.

Dans la littérature critique de ces dernières années, la recherche d'un "antagonisme sérieux à la civilisation déterminée par le capitalisme» se fait sous le terme de "commun", plutôt que sous celui de "communauté». Deux ouvrages récemment parus, celui de Michael Hardt et Antonio Negri, Commonwealth ${ }^{19}$, et celui de Pierre Dardot et Christian Laval, Commun ${ }^{20}$, illustrent cette démarche. Les conceptions du «commun» défendues dans ces deux ouvrages ne sont pas identiques ${ }^{21}$. Elles trouvent cependant l'une et l'autre leur inspiration originaire dans les études consacrées aux communs, c'est-à-dire dans les travaux qui se sont développés depuis plusieurs décennies concernant les différentes formes d'appropriation des biens, et notamment sur la possibilité et la nécessité de distinguer une certaine catégorie de biens dont la nature oblige à dépasser l'alternative

16. J.-L. Nancy, La communauté désavouée, Paris, Galilée (La philosophie en effet), 2014, p. 15.

17. Ibid., p. 31.

18. Ibid., p. 35 .

19. M. Hardt et A. Negri, Commonwealth, E. Boyer (trad.), Paris, Gallimard, 2013.

20. P. Dardot et C. Laval, Commun. Essai sur la révolution au XXI siècle, Paris, La Découverte, 2014.

21. Dardot et Laval discutent et critiquent dans leur livre la conception du commun (ou bien commun) de Hardt et de Negri (voir notamment P. Dardot et C. Laval, Commun..., p. 192-205). - L'ouvrage de Dardot et Laval constitue une somme remarquable sur les théories des communs et du commun. Les positions très élaborées avancées par ces deux auteurs méritent une discussion détaillée que je ne puis faire ici. 
sommaire entre propriété privée et propriété publique (étatique). Les travaux d'Elinor Ostrom, lauréate du prix Nobel d'économie en 2009, ont joué ici un rôle pionnier ${ }^{22}$. Ces théories ont le grand intérêt d'avoir réintroduit dans la pensée de la gauche critique la question de la propriété, centrale chez Marx, mais qui avait été délaissée par les représentants les plus marquants de cette gauche dans la seconde moitié du XXe siècle. Le fait d'user du substantif singulier, «le» commun, plutôt que du pluriel (les communs) plus familier, indique une intention de radicalisation de cette thématique. Hardt et Negri conçoivent le commun, selon la formule par laquelle Dardot et Laval résument la position de ces deux auteurs, comme «le principe philosophique qui doit permettre de concevoir un avenir au-delà du néolibéralisme ${ }^{23}$, eux-mêmes veulent faire de la «constitution politique du commun» une "rationalité alternative [au capitalisme] généralisable ${ }^{24}$. On peut se demander toutefois si les théories «du» commun, sous le couvert d'une radicalisation de la problématique, ne contournent pas la question des régimes d'appropriation des biens, et si elles n'éludent pas du même coup une réflexion sur la fermeture des collectifs politiques, c'est-à-dire sur leurs frontières, un problème qui était au cœur de l'opposition entre théories communautaristes et théories libérales universalistes à la fin du XXe siècle. Cette lacune risque fort d'en limiter l'influence sur les grands débats politiques actuels, largement déterminés ou surdéterminés par les problèmes que pose la gestion des migrations.

\section{Actualité de Max Weber}

Au regard de ces discussions contemporaines, la manière dont Weber traite des communautés, et en particulier des communautés ethniques, de la communauté politique et de la nation, présente à mon sens deux intérêts majeurs. Le premier réside dans sa compréhension résolument non essentialiste des processus de formation communautaire, qui prévient toute interprétation «identitaire» des collectifs sociaux et politiques, quels qu'ils soient (la nation comprise). Le second est l'absence d'opposition entre l'analyse des facteurs socio-économiques et celle des dimensions affectives et symboliques des communautés, c'est-à-dire de ce que l'on vise aujourd'hui

22. À titre d'introduction aux travaux d'Elinor Ostrom, on lira avec profit le numéro 14 de la Revue de la régulation ( $2^{\mathrm{e}}$ semestre, automne 2013), disponible en ligne, https://journals. openedition.org/regulation/10287, et en particulier dans ce numéro l'article de Fabienne Orsi : «Elinor Ostrom et les faisceaux de droits : l'ouverture d'un nouvel espace pour penser la propriété commune» (https://journals.openedition.org/regulation/10471).

23. P. Dardot et C. Laval, Commun..., p. 189.

24. Ibid., p. 156. 
sous le chapeau des «identités», qu'il s'agisse des identités «ethniques", culturelles (liées à la communauté de langue ou de religion, notamment), ou des identités nationales.

\section{La nation}

Weber était, de son propre aveu, un fervent nationaliste. Ce nationalisme s'est exprimé de façon particulièrement virulente dans sa leçon inaugurale à Fribourg en 1895, un texte que même ses plus grands admirateurs éprouvent aujourd'hui quelques difficultés à défendre ${ }^{25}$. Ses positions politiques ont certes bougé entre 1895 et sa mort, en 1920 . Comme il le rappelait luimême en 1917, au début de l'un des plus célèbres de ses textes politiques, "Parlement et gouvernement dans l'Allemagne réorganisée», il avait jadis voté conservateur, puis démocrate, il avait publié autrefois des articles dans la Kreuzzeitung, le journal de l'aile droite du parti conservateur, il le faisait désormais dans des journaux libéraux. Son nationalisme n'était à l'évidence pas le même en ses dernières années que celui de ses années de jeunesse. Mais dans cet article de 1917, il se comptait encore parmi ceux qui placent « fondamentalement les tâches historiques de la nation allemande au-dessus de toutes les questions ayant trait à la forme de son État ${ }^{26}$. Cette allégeance rend d'autant plus remarquable sa capacité exceptionnelle à prendre distance d'avec le nationalisme quand il l'aborde en sociologue, c'est-à-dire à objectiver le nationalisme pour le traiter comme un élément parmi d'autres des facteurs de communautisation.

Dans le manuscrit des Communautés intitulé «Prestige du pouvoir et sentiment national», Weber passe en revue une série de conceptions de la nation pour en montrer, exemples à l'appui, le caractère toujours partiel et insuffisant. La nation ne se confond pas avec le peuple d'un État, c'est-à-dire avec l'appartenance à une communauté politique déterminée (exemple de l'Autriche-Hongrie). Elle ne se confond pas non plus avec une communauté linguistique (exemples de la Suisse, des Serbes et des Croates, des Américains, des Irlandais et des Anglais), même si cet aspect culturel est important.

La prétention à être reconnu comme une «nation» à part est à mettre très régulièrement en relation avec le bien culturel de masse qu'est la communauté

25. Pour ce texte comme pour "Parlement et gouvernement dans l'Allemagne réorganisée», on se référera à la traduction française par un collectif sous la direction d'Élisabeth Kauffmann des plus importants de ses écrits politiques: M. Weber, Euvres politiques (1895-1919), E. Kauffmann et al. (trad.), Paris, Albin Michel, 2004.

26. M. Weber, Euvres politiques..., p. 307. 
de langue (comme c'est massivement le cas dans le pays classique des luttes linguistiques, en Autriche, et aussi en Russie et en Prusse orientale), mais avec une intensité très variable (très faible, par exemple, en Amérique et au Canada) ${ }^{27}$.

La communauté de langue entre notamment en concurrence avec cet autre «bien culturel de masse» qu'est l'appartenance religieuse (exemple des Serbes, orthodoxes, et des Croates, majoritairement catholiques). Que la nation ne repose pas sur une communauté de sang est par ailleurs, selon Weber, quelque chose qui va de soi. Mais l'identité nationale ne peut pas non plus être ramenée à la similitude d'un «type anthropologique» spécifique: le sentiment de partager une identité ethnique, auquel l'un des manuscrits est entièrement consacré, est une représentation (une croyance) qui se nourrit elle-même de sources diverses et hétérogènes. Et ce sentiment lui-même, là où il existe, ne suffit pas pour faire une «nation» (exemple des Polonais de Haute-Silésie). Weber mentionne enfin des couches sociales indifférentes, voire hostiles à l'idée de nation (des couches dirigeantes du prolétariat moderne, jadis les couches féodales, aujourd'hui certaines catégories de la bourgeoisie vivant d'activités «lucratives», etc.) et même des peuples politiques, tels les Luxembourgeois, chez lesquels on ne trouve pas, selon Weber, quelque chose d'équivalent à ce que l'on nomme généralement le sentiment national.

Entre l'affirmation emphatique, le rejet emphatique et, pour finir, la totale indifférence à l'idée de "nation» (qui pourrait, par exemple, être celle des Luxembourgeois, et qui caractérise les peuples qui n’ont pas connu de «réveil» national), conclut-il, il existe tout un éventail d'attitudes très différentes et extrêmement variables, d'une couche sociale à l'autre, et aussi à l'intérieur de chaque groupe qui se voit habituellement qualifier de «nation $»^{28}$.

Le concept de nation s'avère ainsi profondément équivoque, il est, dans la terminologie de Weber, un "concept de valeur», c'est-à-dire une idée à laquelle la plupart des gens attribuent une signification éthique, mais qui ne peut faire l'objet d'une détermination objective.

La «nation» est un concept qui, pour autant qu'il puisse être défini de façon univoque, ne saurait en tout cas l'être d'après les qualités empiriques partagées par ceux qui lui sont rattachés. Pour ceux qui l'utilisent, il ne fait aucun doute qu'il signifie tout d'abord qu'un sentiment spécifique de solidarité ne peut qu'être attendu de certains groupes humains face à d'autres, ce qui inscrit

27. M. Weber, Les communautés, p. 197.

28. Ibid., p. 199. 
ce concept dans la sphère de valeurs. Mais il n'existe aucun consensus sur la façon dont ces groupes doivent être délimités ou sur les modalités de l'activité communautaire censée résulter de cette solidarité ${ }^{29}$.

\section{Gemeinsamkeit}

J'ai évoqué plus haut les différences entre l'appareil conceptuel des Communautés et celui des Concepts fondamentaux. En dépit de ces différences, la façon dont Weber appréhende les communautés est, dans le texte plus ancien, déjà très clairement dynamique. Cette approche permet d'éviter les réifications identitaires des phénomènes communautaires. Les passages que je viens de citer et de résumer concernant la nation sont exemplaires de la manière dont il procède: il soumet chaque type de communauté étudiée (domestique, ethnique, politique, nationale, etc.) à l'épreuve d'exemples historiques multiples, ce qui lui permet d'en montrer dans chaque cas le caractère labile, fonction d'éléments certes objectifs, pour partie du moins (la langue commune, des coutumes et modes de vie semblables, la confession religieuse, etc.), mais dont aucun n'est suffisant pour fonder une communauté. Tous ces éléments sont susceptibles de fonctionner comme facteurs de communautisation, c'est-à-dire de favoriser le développement d'une activité commune, mais aucun ne peut être considéré comme suffisamment important pour expliquer à lui seul l'existence d'une communauté déterminée. Significatif est à cet égard l'absence dans le lexique wébérien du terme "identité», ou d'un terme ayant la même signification.

Il est cependant un terme fréquemment utilisé dans les Communautés, Gemeinsamkeit, qui, dans certaines de ses occurrences, peut être rendu par «identité». "Communauté» eût été aussi une traduction possible (quand il est question par exemple de communauté d'origine, de communauté linguistique ou de confession religieuse) si le terme n'était pas déjà préempté pour la traduction de Gemeinschaft. Weber lui-même a explicitement souligné la différence de signification entre les deux termes, tels qu'il les utilise, dans un passage du manuscrit sur les communautés ethniques.

Le lien commun [Gemeinsamkeit] «ethnique» se distingue de la «communauté de clan " précisément en cela qu'il n'est en soi qu'un «lien commun " (auquel l'on croit), et non une "communauté» (comme l'est le clan), dont l'essence implique une activité communautaire réelle. Le lien commun ethnique (au sens ici visé), en revanche, n'est pas lui-même une communauté, mais seulement un élément qui facilite la communautisation ${ }^{30}$.

29. M. Weber, Les communautés, p. 196.

30. Ibid., p. 139-140. 
On trouve une remarque similaire, qui en élargit la portée (il ne s'agit pas seulement des communautés ethniques), dans les Concepts fondamentaux de sociologie (II, $\$ 9$, remarque 4 ):

Le fait de partager [Gemeinsamkeit] des qualités, des situations ou des comportements communs ne saurait nullement constituer une communauté. Par exemple, le fait de partager un même patrimoine biologique héréditaire, considéré comme un caractère "racial», ne signifie pas à soi seul, bien évidemment, une quelconque communautisation des individus ainsi distingués ${ }^{31}$.

Que cette distinction soit faite à propos des communautés ethniques n'est pas un hasard: la croyance en une origine ethnique commune, voire à une identité raciale ${ }^{32}$, est un terrain particulièrement favorable à la réification des communautés. On voit ici que Weber était solidement prémuni contre les conceptions raciales de la communauté que les idéologues du nazisme ont propagées dans les années qui ont suivi sa mort. Les pages qu'il consacre aux communautés "ethniques» constituent en vérité une déconstruction de la notion. Elles se terminent en effet sur ce constat:

$\mathrm{Au}$ total, nous trouvons réunis dans l'activité communautaire conditionnée «ethniquement» des phénomènes qu'une analyse sociologique vraiment exacte [...] devrait séparer soigneusement. [...] [Tous ces phénomènes] devraient être analysés en détail et séparément. Ce faisant, on jetterait certainement totalement par-dessus bord le concept collectif de l'«ethnique». Car c'est un terme collectif parfaitement inutilisable pour toute analyse vraiment exacte ${ }^{33}$.

Et encore: «Le concept de communauté "ethnique" se volatilise pour qui s'attache à une conceptualisation exacte " ${ }^{34}$, ce qui, ajoute-t-il, le rapproche de celui de nation.

La distinction entre Gemeinschaft et Gemeinsamkeit a cependant, je l'ai dit, une portée plus générale. Encore une fois: aucun trait commun, qu'il s'agisse de l'apparence physique, de la langue, de la religion, etc., n'est à lui seul suffisant pour fonder une communauté. Les identités collectives (si on tient à conserver ce terme, et il est sans doute difficile de s'en passer) sont toujours des identités représentées (des phénomènes de croyance,

31. M. Weber, Concepts fondamentaux..., p. 144.

32. Concernant les positions de Weber à propos de la question, à l'époque très discutée, des déterminations biologiques des comportements sociaux, voir E. Winter, Max Weber et les relations ethniques. Du refus du biologisme racial à l'État multinational, Québec, Presses de l'Université Laval, 2004.

33. M. Weber, Les communautés, p. 149.

34. Ibid. 
dans la terminologie de Weber). Ces représentations peuvent s'appuyer sur des éléments objectifs (la langue, les phénotypes, la religion, les modes de vie, etc.). Mais l'importance que des groupes d'individus attribuent à ces éléments objectifs dans leur différenciation d'avec d'autres groupes est fonction de configurations toujours singulières auxquelles contribuent de nombreux autres facteurs possibles de communautisation. Le fait d'appréhender les communautés du point de vue dynamique de leur formation implique de prendre en compte la pluralité des facteurs qui se composent, se confortent, mais qui peuvent aussi jouer les uns les autres. La stabilité d'une communauté constituée n'est pour cette raison jamais assurée: il existe toujours en son sein des facteurs qui tendent à remettre en question son unité et ses frontières.

\section{La nation et l'État: Weber ou Mauss?}

Parmi ces divers facteurs, Weber accorde une attention particulière au «sentiment de prestige» qu'une collectivité politique peut tirer de l'affirmation de sa puissance dans ses rapports avec d'autres communautés de même type. Ces remarques, essentiellement inspirées par l'État-nation moderne (même si elles peuvent être étendues aux empires), permettent une comparaison intéressante avec le texte de Marcel Mauss sur la nation ${ }^{35}$. Cette comparaison montre tout d'abord que le fait d'appréhender la nation, non comme une entité fixe, mais dans la perspective dynamique de son procès de formation, n'est pas totalement exceptionnel, dans les premières décennies du $\mathrm{XX}^{\mathrm{e}}$ siècle déjà. Comme le soulignent les éditeurs dans leur présentation du texte de Mauss, celui-ci «ne perçoit pas la nation comme une chose statique, mais comme un déroulement historique, comme un processus. De manière similaire à Simmel et Weber, Mauss conçoit la société comme une réalité complexe et en mouvement, ce qui le conduit à proposer une théorie de la nationalisation plus qu'une théorie de la nation ${ }^{36}$. Il est cependant une différence entre l'approche wébérienne et celle de Mauss. Ce dernier fait de l'État national, et plus précisément de l'État de droit, voire de l'État démocratique, le telos des processus de nationalisation, ce qu'indiquent par exemple son emploi des expressions de «nation complète» ou de "nation achevée», ainsi que ses développements sur les sociétés africaines, certains États d'Amérique latine et le

35. M. Mauss, La nation, M. Fournier et J. Terrier (éd. et prés.), Paris, PUF (Quadrige), 2013. - Il s’agit d'un manuscrit conçu par Mauss au début des années 1920, qu'il n’a jamais achevé ni publié.

36. M. Mauss, La nation, p. 24-25. 
Japon, qui ne sont «pas encore» de véritables nations. Si l'on s'en tient aux positions politiques de Weber et de Mauss, on pourra préférer le plaidoyer du second pour un internationalisme garant de la paix entre les nations à la conviction wébérienne selon laquelle le prestige du pouvoir est un trait caractéristique, et peut-être indépassable, des grands États, ceux dont dépend selon lui l'avenir de la civilisation mondiale. On pourra aussi, dans la perspective des historiens, expliquer cette différence, pour partie du moins, par l'écart chronologique qui sépare les deux auteurs: avant ou après la Première Guerre mondiale. La pensée politique de Weber s'est formée dans les dernières années du XIX ${ }^{\mathrm{e}}$ siècle, elle s'est prolongée durant le conflit de 1914-1918, ses derniers écrits commentent l'effondrement de l'Allemagne, à laquelle les pays vainqueurs ont imputé l'entière responsabilité de la guerre et imposé le «diktat» du traité de Versailles. L'ouvrage (inachevé) de Mauss a été conçu après guerre (au début des années 1920), son auteur soutient le projet de pacification des relations internationales porté par la Société des Nations. Mais la raison profonde des différences entre les deux auteurs, s'agissant des rapports entre État et nation, tient plus profondément à leurs conceptions respectives de la sociologie. Mauss partage certes avec Émile Durkheim, dont il était le neveu et le disciple, un rejet des abstractions normatives de la philosophie et la volonté de constituer la sociologie (qui deviendra chez lui une anthropologie sociale) en une science positive fondée sur des études empiriques. Mais il hérite aussi de Durkheim l'idée que cette science sociale positive a une vocation «morale», en ce sens qu'elle est en mesure de justifier directement des propositions de réformes politiques. Cette vocation morale attribuée à la sociologie explique les ambiguités et les limites de son texte sur la nation ${ }^{37}$, et notamment qu'il puisse parfois, dans ses tentatives de définitions de la nation, inclure parmi les éléments constitutifs de celle-ci, à côté de l'adhésion consciente à une collectivité politique, des éléments objectifs tels qu'une «race», une «langue», une «morale», un « caractère national ${ }^{38}$. Cette confusion entre l'étude empirique et les visées normatives fait ressortir par contraste l'intérêt de la «neutralité axiologique» qui sert de règle à la pratique wébérienne de

37. Les éditeurs du texte en sont parfaitement conscients, comme l'indique le titre de leur préface: «La nation: une expédition dans le domaine du normatif».

38. Ce passage, notamment, motive la critique que Rogers Brubaker fait des positions de Mauss sur la nation (voir R. Brubaker, «Marcel Mauss on Nationhood: Objectivism and its Limits", in Studies on Nationalism, M. Kovács et P. Lom [dir.], Budapest, Central European University Press, 2004, p. 105-114). Marcel Fournier et Jean Terrier ne manquent pas de le citer, mais ils invitent à en relativiser la portée, de même que Bruno Karsenti (voir B. Karsenti, «Une autre approche de la nation: Marcel Mauss », Revue du MAUSS, ${ }^{\circ}$ 36, 2010, p. 283-294). 
la sociologie. Weber est sans doute plus «nationaliste» que Mauss, mais, quand il s'affronte au matériel historique-empirique et tente d'élucider les concepts avec lesquels en rendre compte, il suspend toute préconception de ce que la nation doit être. C'est à juste titre que Rogers Brubaker a pu écrire, dans un article datant de 2004, que «[1]es brèves discussions de Max Weber concernant l'ethnicité et le nationalisme dans Économie et société sont parmi les pages les plus pénétrantes qui aient jamais été écrites sur le sujet, anticipant les formulations constructivistes sophistiquées des deux dernières décennies ${ }^{39}$. Son appréciation de la position de Mauss sur la nation (qui est l'objet principal de l'article cité) est plus réservée.

\section{La dimension économique des phénomènes communautaires}

Il est un second aspect des textes de Weber sur les communautés qui présente un intérêt du point de vue des thématiques contemporaines, à savoir l'importance centrale qu'il accorde à la dimension économique des processus de formation des communautés. Le premier manuscrit porte sur «les relations économiques des communautés en général». La place centrale accordée à l'économie est une caractéristique générale de la sociologie wébérienne. Elle rend nécessaire, à l'époque où écrit Weber, une prise de position explicite à l'endroit de la conception matérialiste de l'histoire. Weber le fait à différentes reprises, et en particulier au début du texte des Communautés. Contre le «monisme économique» qu'il reproche au marxisme de son temps, il défend un pluralisme causal et multidirectionnel, selon lequel les différentes dimensions des activités humaines ne sont pas indépendantes les unes des autres mais se conditionnent réciproquement. Chacune de ces dimensions possède cependant une logique qui lui est propre, de telle sorte que le postulat d'une détermination en dernière instance de l'ensemble des phénomènes sociaux par l'une ou l'autre de ces dimensions, et en particulier par l'économie, fait inévitablement violence à la réalité historique.

La centralité de l'économie dans les travaux sociologiques de Weber peut donc être considérée comme le choix d'un point de vue particulier sur les phénomènes sociaux. Il est un passage bien connu de l'Essai sur l'objectivité dans lequel Weber théorise cet aspect constructiviste de la définition de l'objet de la sociologie ${ }^{40}$.

39. "Max Weber's brief discussions of ethnicity and nationalism in Economy and Society are among the most penetrating pages ever written on the subject, anticipating the sophisticated constructivist formulations of the last two decades" (R. Brubaker, "Marcel Mauss on Nationhood...", p. 105; je traduis).

40. J'ai moi-même souvent utilisé ce passage pour soutenir que la centralité de l'économie dans la sociologie wébérienne relève d'une orientation de l'intérêt (autrement nomméle «rapport 
Le point de vue dit «social», écrit-il, c'est-à-dire celui de la relation entre les hommes, ne possède vraiment une précision suffisante pour délimiter les problèmes scientifiques qu'à la condition d'être accompagné d'un prédicat spécial quelconque déterminant son contenu ${ }^{41}$.

La lecture attentive des manuscrits sur les Communautés laisse penser cependant que l'économie n'est pas, aux yeux de Weber, un "prédicat» quelconque. Le premier de ces manuscrits, «Les relations économiques des communautés en général», s'ouvre sur un constat: "Dans leur écrasante majorité, les communautisations ont des relations, quelles qu'elles soient, avec l'économie » ${ }^{42}$. Cette proposition est suivie d'une définition de l' «économie», définition large, qui n'est certainement pas «marxiste», mais qui n'est pas non plus inspirée par l'économie marginaliste, malgré la référence que Weber fait au «besoin» et malgré la similitude entre la définition de l'économie qu'il propose ici et celle qu'il donnait dans ses cours de la fin des années 1890 à Fribourg et Heidelberg. On passera sur les distinctions que Weber introduit ensuite entre une communauté économique proprement dit, une communauté ayant une activité économique, une communauté régulatrice de l'économie, qui seront reprises dans la première entrée des "Catégories sociologiques fondamentales de l'économique » 43 (à ceci près que Weber n'y parle plus de communauté, mais seulement d'activité). Plus décisive, pour comprendre les relations entre les différentes formes de communautés et l'économie, est l'idée selon laquelle on peut parler d'économie dès lors que des individus ou des groupes d'individus sont en concurrence pour l'appropriation de biens (matériels ou idéels) qui ne sont pas disponibles en quantité illimitée:

En ce qui nous concerne [...] nous entendons bien plutôt ne parler ici d'économie que là où, face à un besoin ou un ensemble de besoins, il existe en comparaison, d'après ce qu'estime celui qui agit, un stock très restreint de moyens et d'actions possibles pour les couvrir, et où cette situation induit un comportement qui en tienne spécifiquement compte ${ }^{44}$.

La notion d'«appropriation» apparaît à diverses reprises dans les Communautés, avant tout dans les pages du premier manuscrit qui traitent

aux valeurs", Wertbeziehung), certes indispensable pour déterminer des questionnements spécifiques, mais qui ne préjuge pas d'une centralité objective de l'économie dans les procès de socialisation. Cette interprétation me parait aujourd'hui forcer le "constructivisme» de Weber.

41. M. Weber, Essais sur la théorie de la science, J. Freund (trad.), Paris, Plon (Recherches en sciences humaines), 1965, p. 145-146.

42. M. Weber, Les communautés, p. 61.

43. M. Weber, Économie et société I, p. 62.

44. M. Weber, Les communautés, p. 62. 
des «relations économiques "ouvertes" et "fermées" »45. Les "Catégories sociologiques fondamentales de l'économique» consacreront toute une série d'alinéas à une différenciation casuistique entre diverses formes d' «Appropriation" ${ }^{46}$, dont il n'est pas d'équivalent dans les Communautés. Malgré cela, pour qui lit la totalité des Communautés, il apparait que c'est bien l'appropriation de biens (matériels ou idéels, des "chances») qui constitue le point d'articulation principal entre la dimension économique des processus de communautisation et leurs autres dimensions, notamment ces éléments que l'on range aujourd'hui du côté des identités symboliques et affectives (religieuses, culturelles, nationales, etc.). Les procès de communautisation tendent en effet à la fermeture: une communauté ne prend véritablement consistance que lorsqu'elle se distingue et s'oppose - de manière conflictuelle ou pas - à d'autres communautés de même nature. Ce qui rend nécessaire cette fermeture, ce qui en est l'enjeu, est l'appropriation monopoliste de certains biens au profit des membres de telle ou telle communauté déterminée. Il est un passage du premier manuscrit qui me paraît décisif à cet égard:

Un mode de conditionnalité économique qui se rencontre très fréquemment dans toutes les formes de communautés est créé par la concurrence pour des chances économiques: charges administratives, clientèle, occasion de s'emparer d'un bien ou de trouver du travail, etc. À mesure qu'augmente le nombre des concurrents par rapport à l'espace ouvert pour l'acquisition, augmente aussi l'intérêt que ceux qui participent à la concurrence ont à limiter celle-ci d'une manière ou d'une autre. Cela se produit généralement sous la forme suivante: une caractéristique quelconque d'une partie des concurrents (actuels ou potentiels) qui peut être constatée extérieurement - la race, la langue, la confession, l'origine géographique ou sociale, l'ascendance, le lieu de résidence, etc. - est prise par les autres comme prétexte pour tenter de les exclure de la concurrence. Ce qu'est cette caractéristique dans chaque cas singulier est indifférent: elle est associée à chaque fois au premier élément qui s'offre. L'activité communautaire qui s'est ainsi formée chez les uns peut alors susciter une activité correspondante des autres, contre lesquels elle est dirigée ${ }^{47}$.

Weber parle certes d'un mode de conditionnalité économique des communautés. Ce n'est pas le seul, bien entendu, et l'angle de lecture que je propose ici n'épuise pas toutes les ressources analytiques qui peuvent se tirer de ces textes. Comment le pourrait-il, quand Weber lui-même remarque, à propos du rapport entre l'économie et les formes structurelles de l'activité

45. M. Weber, Les communautés, p. 66-69.

46. Voir M. Weber, Économie et société I, p. 128-139.

47. M. Weber, Les communautés, p. 66 (je souligne). 
communautaire, qu'il est impossible de «rien dire de totalement général qui soit intéressant ${ }^{48}$ ? On doit se contenter, ajoute-t-il dans la foulée, de constater des "affinités électives» entre certaines formes structurelles concrètes d'activités communautaires et des formes concrètes d'économie, et de formuler quelques thèses générales sur la manière dont des intérêts économiques induisent certains types d'activité communautaire. Si j'insiste cependant sur cette forme de "conditionnalité» qu'est l'appropriation tendanciellement monopolistique de «chances» déterminées par une communauté, c'est, tout d'abord, parce que, à la lecture de l'ensemble des Manuscrits, on constate qu'elle joue un rôle décisif dans la formation des communautés ainsi que dans leurs différenciations internes (entre classes ou groupes statutaires). Mais c'est aussi parce qu'elle permet d'aborder la question des nécessaires ou inévitables «fermetures» communautaires, et par là même de leurs frontières.

Il importe peu, de ce point de vue, que l'on parle en termes de «communautés» ou de «relations sociales». Les Concepts fondamentaux de sociologie, texte dans lequel Weber fait de la «relation sociale» et non plus de la «communauté» la clé de voûte de sa sociologie, comportent également une entrée $(\$ 10)^{49}$ sur les «relations sociales ouvertes et [...] fermées», qui reprend les éléments d'analyse que l'on trouve dans le passage correspondant des Communautés. Rarement sollicité, à ma connaissance, dans les travaux d'exégèse wébérienne, ce texte lie les processus de fermeture des relations sociales à la tendance, inhérente à toute forme d'appropriation, à monopoliser des «chances" de diverses natures au profit d'un groupe limité d'individus. Il débouche sur une compréhension originale de la «libre propriété» (freies Eigentum), interprétée comme une spécification de la propriété privée (Eigentum) qui subvertit les conditions communautaires qui sont en général celles de l'appropriation. À l'encontre des théories philosophiques de la propriété, celles des XVII et XVIII siècles et d'aujourd'hui encore, qui visent à justifier d'un point de vue normatif l'appropriation privative individuelle par rapport à un commun indifférencié, Weber conçoit la propriété privée libre comme la limite extrême d'un processus d'appropriation qui est à l'origine toujours collectif (par des communautés déterminées). Cette limite signifie cependant un renversement: la possibilité pour un individu d'aliéner sans condition les biens qu'il possède est à la fois le point ultime des processus de fermeture «intérieure» des communautés (c'est-à-dire de la monopolisation de l'accès à certaines catégories de chances par des fractions d'une communauté plus large) et l'abolition

48. Ibid., p. 65

49. M. Weber, Concepts fondamentaux..., p. 146-150. 
des conditions communautaires de l'appropriation. Cette interprétation très originale des conditions de formation de la propriété privée libre n'est qu'indiquée dans les Concepts fondamentaux ${ }^{50}$. Elle est plus précise dans un passage des Communautés, que je me permets de citer assez longuement, laissant au lecteur d'apprécier l'usage que l'on pourrait en faire pour rendre compte des effets corrosifs que le capitalisme néolibéral et la montée en puissance du capitalisme financier exercent sur les solidarités collectives:

La libération complète des chances de monopole appropriées pour qu'elles puissent être échangées, y compris vers l'extérieur, c'est-à-dire leur transformation en propriété [Eigentum] complètement «libre», signifie naturellement que vole en éclat l'ancienne communautisation monopolisée. Ce qui reste, à titre de caput mortuum, de cette communautisation, ce sont des pouvoirs de disposition, désormais appropriés en tant que «droits acquis», détenus par les individus dans le commerce des biens. Car, sans exception, toute «propriété» [Eigentum] sur des biens naturels s'est formée historiquement à partir de l'appropriation progressive des parts que des compagnons avaient à des monopoles $[\ldots]^{51}$.

\section{Conclusion}

Quelle attitude la pensée critique peut-elle ou doit-elle adopter à l'endroit des notions de communauté ou d'identité, et en particulier de communauté et d'identité nationale? Est-il juste de reprocher à la pensée critique de s'être trop exclusivement focalisée sur l'économie et le social, au détriment des dimensions affectives et symboliques des appartenances collectives?

Max Weber ne peut certes être annexé à ce que l'on nomme aujourd'hui la "pensée critique», pour autant que celle-ci, qu'elle se réfère ou non explicitement au marxisme, tend à mettre fondamentalement en question la rationalité des structures économiques et politiques des sociétés modernes. Sa conception intransigeante des conditions qui sont celles de l'objectivité scientifique dans le domaine des sciences sociales et politiques, à savoir la suspension par le savant, économiste, sociologue ou autre, de tout jugement normatif (le principe dit de la "neutralité axiologique»), est généralement mal comprise par ceux qui s'en réclament ${ }^{52}$. D’autres

50. M. Weber, Concepts fondamentaux..., p. 145.

51. M. Weber, Les communautés, p. 68.

52. C'est le cas des auteurs qui invoquent ce principe pour dénoncer l'idée même d'une sociologie critique (voir Le Débat, $n^{\circ}$ 197, 2017, p. 113-141, ainsi que G. Bronner et E. Géhin, Le danger sociologique, Paris, PUF, 2017). Sous le couvert de l'objectivité scientifique, ils visent en vérité à une véritable censure des objets légitimes de la sociologie. Qu'ils comptent le pouvoir, le capitalisme, la domination, les inégalités, etc., parmi les objets suspects, témoigne de leur 
la tiennent pour impossible et considèrent que Weber lui-même enfreint souvent ce principe dans nombre de ses propres analyses. Sans pouvoir ici reprendre ce débat, on notera que la manière dont le nationaliste Weber traite du nationalisme dans le texte des Communautés montre que cette suspension des options éthiques et politiques est possible, et qu'elle n'est pas sans portée critique. À défaut de nous fournir des points de référence pour des jugements normatifs, le prosaïsme des analyses wébériennes met en lumière la part d'ombre inévitable dans toute appréhension du social commandée, explicitement ou implicitement, par une idée préconçue de ce que les sociétés peuvent ou doivent être. Cette lucidité l'immunise tout autant contre les aveuglements des apologètes de l'existant que contre ceux des radicalismes révolutionnaires. Loin d'être un obstacle pour une sociologie critique, cette pratique de l'objectivité scientifique peut être considérée au contraire comme une forme accomplie de la pensée critique.

Pour conclure, et au risque de me répéter, je résume ici les deux aspects des analyses des Communautés qui me paraissent particulièrement propres à éclairer certains débats actuels.

On a vu tout d'abord que ces analyses proscrivent toute interprétation substantielle, liée à des caractères «objectifs», des identités collectives. S'agissant plus précisément de la nation, Weber déploie les éléments très divers qui contribuent à forger ce sentiment d'appartenance collective spécifique que nous nommons aujourd'hui la nation. L'existence d'une communauté politique, à l'époque moderne un État, est un de ces éléments, et Weber, on l'a dit, lui accorde une importance particulière. Malgré cela, il n'identifie par État national et nation, et ne considère pas non plus que l'idée de nation trouve son accomplissement dans l'État national. Du point de vue de la compréhension sociologique, il n'est nulle raison de faire un sort à part à une conception purement politique, pas même à une conception républicaine de la nation. Elle est, elle aussi, une représentation qui ne peut épuiser la signification de la notion et elle peut, tout comme d'autres, prêter à des fixations identitaires.

En second lieu, à l'encontre de l'opposition souvent faite aujourd'hui entre les déterminations économiques et les dimensions affectives et symboliques des appartenances collectives, Weber montre que les unes et les autres sont étroitement imbriquées dans la formation et la persistance des communautés. Directement ou indirectement, les phénomènes communautaires ont toujours pour enjeu (même si ce n'est pas le seul) l'appropriation de biens ou d'opportunités diverses au profit de groupes

profonde ignorance de l'œuvre de Max Weber. Que resterait-il en effet de cette œuvre si l'on en supprimait toutes ces catégories? 
déterminés. L'idée, explicite ou implicite chez les théoriciens $d u$ commun, selon laquelle le monde pourrait être organisé de telle sorte que les frontières communautaires tendraient à disparaître, apparaît de ce point de vue illusoire. La concurrence pour l'appropriation monopolistique de "chances» déterminées (biens, matériels ou idéels) par des groupes spécifiques est une dimension structurelle des phénomènes communautaires qu'une conception réaliste de la politique ne doit pas ignorer. Son abolition est une utopie: les communautés sont impensables sans frontières (spatiales ou autres).

Mais il est aussi faux, et politiquement dangereux, d'attribuer la résistance des fermetures communautaires, et en particulier, aujourd'hui, des idéologies nationalistes, à la prévalence du symbolique dans les identités collectives. Les éléments qui contribuent à la formation des communautés sont multiples et hétérogènes, certains sont objectifs (langue, religion, histoire politique partagée), d'autres ne sont que des «croyances subjectives». L'importance qui revient à chacun de ces éléments dans les synergies communautaires est fonction de conjonctures toujours singulières. N'importe quel trait commun (Gemeinsamkeit) - «le premier qui s'offre", selon l'expression de Weber -, qu'il soit objectif ou imaginaire, peut servir de justification symbolique à la monopolisation de certaines catégories de chances par un groupe déterminé. Il est cependant essentiel d'insister sur le fait que ce n'est pas un caractère commun (Gemeinsamkeit), quel qu'il soit, qui fait communauté, mais l'activité partagée. Reconnaître l'importance du facteur économique de l'appropriation dans les processus communautaires n'interdit pas en effet de tenter de préserver certaines catégories de biens (les communs) de l'appropriation exclusive (et en particulier de la privatisation), ni d'œuvrer en faveur de l'assouplissement des conditions d'accès à des biens qui ne peuvent exister sans fermeture communautaire (par exemple les protections sociales garanties par un État). Et c'est en outre une tâche urgente aujourd'hui que d'empêcher que les logiques monopolistiques des appropriations communautaires, en particulier dans leurs déclinaisons nationalistes, n'aboutissent à ce que certains - voire des masses considérables de populations, migrants et miséreux - soient définitivement exclus de la concurrence pour les biens de ce monde. 